\title{
47 \\ Simulators: Tools for Teaching Theory of Computation
}

Tiarajú A. Diverio, Ingrid V. Mito, Thiago F. Moesch and Luis F. R. Lima Instituto de Informática e PPGC da UFRGS, C.P. 15064, 91501-970 Porto Alegre, BRAZIL diverio@inf.ufrgs.br

Keywords: higher education, computation, simulation, distance learning, tutor

\begin{abstract}
This paper describes simulators, which are programming tools that make available the following formalisms: Turing Machine, Register Machine and Post Machine. With these simulators it is possible to teach Theory of Computation in a new way, where students may visualise numerical computed functions and check language recognition, including, therefore, the chapters of computability and universal machines. These simulators are part of the project Theory of Computation Laboratory. Also, this project consists of a set of educational software simulators built to improve teaching with quality and provide tools for the remote teaching project. The objective of this research is to develop software that gives more flexibility for its users in editing and creating abstract machines, by providing a friendly user interface, large set of operations, and knowledge base of these machines. This is the foundation for an integrated teaching environment on the Web.
\end{abstract}

\section{INTRODUCTION}

The motivation for this work was the lack of educational software for teaching theoretical computations, and also the importance of generating qualified human resources to work in the several computer graduation courses existent in Brazil. This work is meant to help students, through 
simulated programs, to understand the computational formality studies in the Theory of Computation subject including the Turing Machine, Finite Automatons, Post Machine, Norma Machine, and Lambda Calculus. Through simulators we can create and carry out programs related to the desired machine in a fast, simple and intuitive way. The system aims to help not only students in understanding the content, but also teachers, who will have in their hands a powerful tool for creating exercises, their correction and demonstrating examples in classrooms. This paper proposes that simulators are important tools for teaching in general.

\section{SIMULATORS}

Simulators are instructional packages that make available the visualisation of formalisms or theoretical machines such as: Turing Machine, Norma Machine, Finite Automatons with Stacks, and Post Machine (Diverio and Franciosi 1996, Mito and Diverio 2001, Moesch et al. 1998).

The objectives of these simulators are the development of a laboratory environment for the students that are Bachelors in Computer Science of the Federal University of Rio Grande do Sul (UFRGS). Here students can develop programs in different machines, run programs step by step for learning and correction, solve exercises, and provide assistance for teachers in the working out and correction of exams.

The idea of the simulators arose during a practical task carried out in the discipline of Theory of Computation. Due to the good quality of the works presented, it was decided to develop a project to make instructional packages available in a local environment (the laboratories of the Computer Science Institute of UFRGS). So some alterations were necessary in order to transform the different prototypes into a unique environment. The alterations aim at standardisation and unification of the interface and uniformity in the computational environment (language and computer). For standardisation the following is defined.

- The simulators are developed in Delphi through the Pascal language Oriented to Object. The system's operation platform is based on PC's with operational system Windows.

- All simulators create files that can be saved on disk for further reading. All files are saved in ASCII format, using a simple structure making them portable to any architecture and accessible to other modules.

- The simulators have a standard installer interface of the programs compatible with Windows. In this case, it was used the InstallShield ${ }^{T M}$ as an installer software, which will copy the files and offer a help system. 
- The Execution can present the final result of the computation, and also the possibility of carrying out the computation process step by step, so that the user can interpret the results in due time. Two ways of running have been defined: Uninterrupted (the alterations performed at each step of the computation are showed, but the execution only stops when it is finished), and Step-by-Step (the execution stops at each step of the computation, and waits until the user authorises the continuation of the process).

- The simulators count on a help system that assists the user during the utilisation of the software. In this system it is also included the idea of tutor, which gives concepts about the simulator in use.

Three simulators developed are described in the next sections.

\section{TURING MACHINE SIMULATOR}

Among all the universal machines studied, the Turing Machine is notably the most important because it is simple and powerful. The Turing Machine consists basically of a one-side infinite tape, a tape head and a program. The tape head has the capacity of reading and writing a symbol on the tape and, after that, according to the program's specification, moving a cell to the left or a cell to the right. A complete definition of the Turing Machine can be found in Diverio et al. (2000).

The Simulator of the Turing Machine is composed of four modules: tutor, program editor, program emulator through graphs, and program emulator through functions. The Tutor is composed of the basis of knowledge about the chapter on computability through Turing Machines, which can be accessed in an electronic book or help format. In the current stage, the Tutor is not equipped with intelligence, but studies are being developed with this purpose in mind.

\subsection{Program emulator through Graphs}

The emulator programs through Graphs allow the definition of programs by a state machine. Among the available functions in this tool we have the definition of a Turing Machine (in which the entry alphabet, state set, auxiliary alphabet, final state sets, initial state and program are introduced), editing of machine already defined, saving of machine in file and running of a program. For the definition there are available tools such as editing, state creation, indicator of the initial and final states, editions of transitions. In Figure 1 we showed a transition form, where $p$ is the concurrent state, $q$ is 
the new state, $a_{n}$ is the read symbol, $a_{v}$ is the writing symbol, and ' $m$ ' the movement of the head, which can be to the left or to the right.

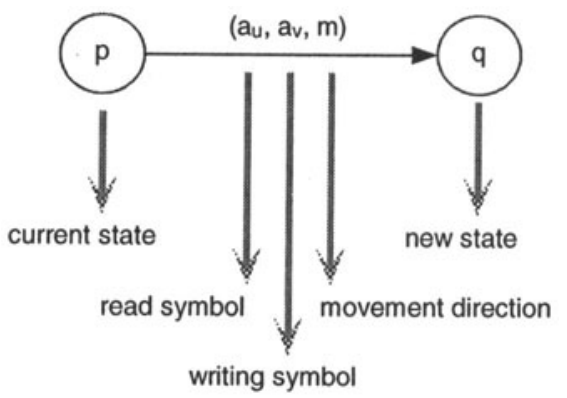

Figure 1. Definition of transition through graphs

For the edition of a transition, the program offers a window (Figure 2) with the pre-defined values. Operations with states and transitions are available; states and transitions can be removed. The interface offers a tool bar that is activated by the $T$ icon. The graph can be easily re-ordained when moving the states. At the end of the edition we can save, create an image of the graph and insert this image in other file.

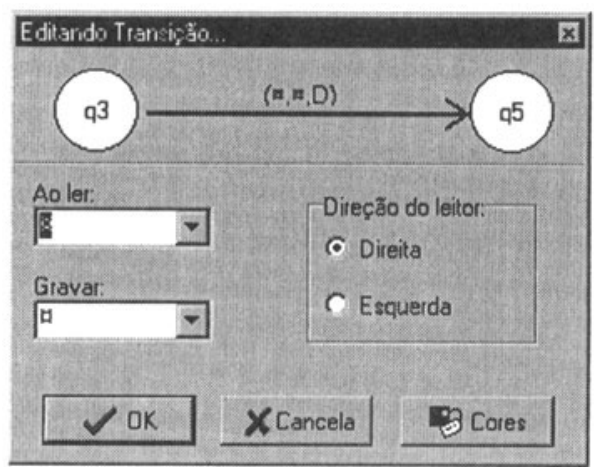

Figure 2. Edition Screen

Once the consistence of the program is achieved, we can execute it by clicking the execution icon. The tape will appear all filled in by the special symbol on a white character $(\beta)$ and also a window to introduce the entry. The execution can be done in the two formats: step by step or uninterrupted. In both cases the current position in the tape and the current state will be displayed as they are coloured. Figure 3 shows the screen of the program 
emulator through graph. The graph showed in that figure performs the parenthesis evaluation.

So, to sum up, this module describes the program as a graph in which the vertices represent the states and transitions, which may involve change of symbol, displacement and move of state. It is characterised by the creation of automatons in a fast and intuitive way. Therefore, the user is provided with a tool bar with simple functions such as create a state, create a transition, edit a transition and other functions.

The states can be positioned on the screen according to the user's preferences, by just moving it with the mouse. It allows the re-drawing of the transitions, considering their new positions. The alteration of the states can be given through the tool bar or through a click on the right button of the mouse. The transitions also can be modified in a very simple way, and just two clicks are necessary on the desired transition.

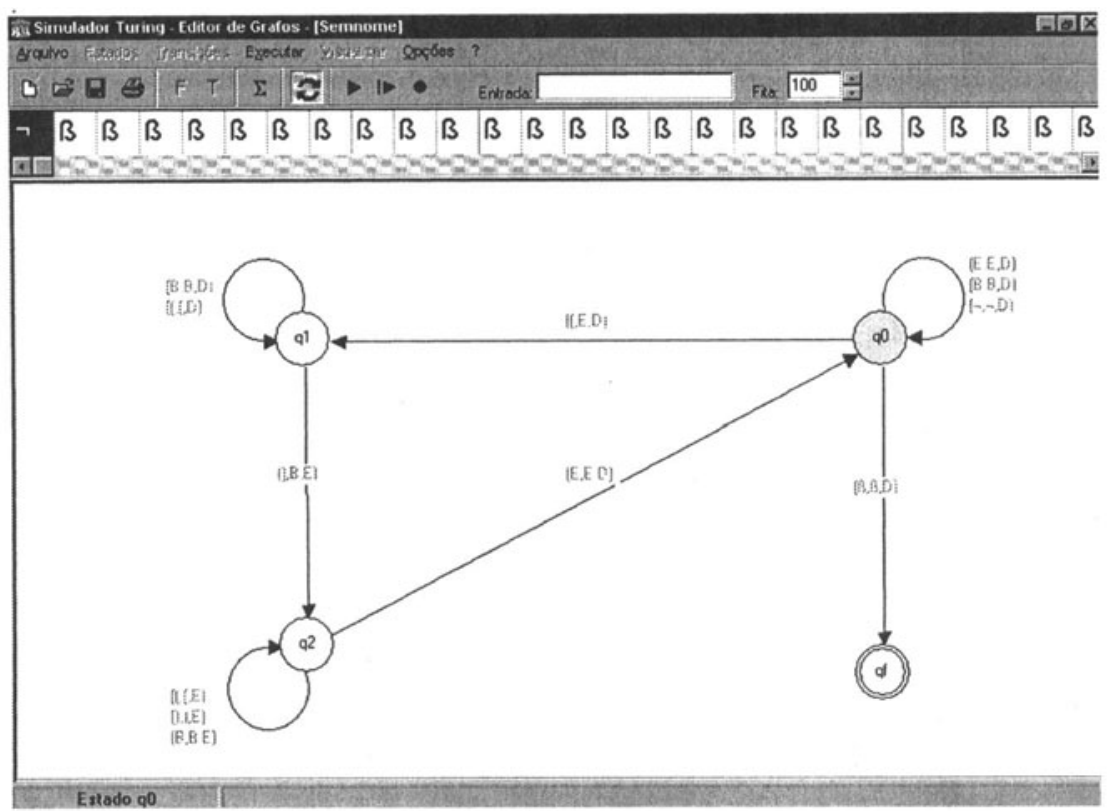

Figure 3. Program Emulator through graph's screen

\subsection{Program emulator trough transition functions}

The program emulator through transition functions implements the definition that uses a matrix of two states in order to represent the program, 
and offers resources such as copy and paste. In Figure 4, the same example of the Turing Machine is presented, which performs the parenthesis evaluation. We can notice in Figure 4 that, during the execution, the current symbol of the tape is selected, as well as the matrix field that is being analysed. The software allows the user to dimension again the size of the lines and columns of the matrix, as well as alter its style and its font size.

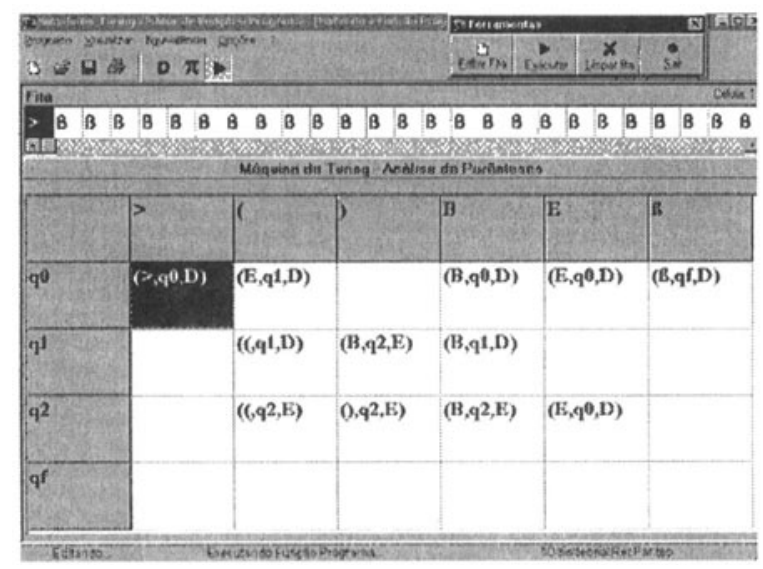

Figure 4. Turing Machine that evaluates parenthesis

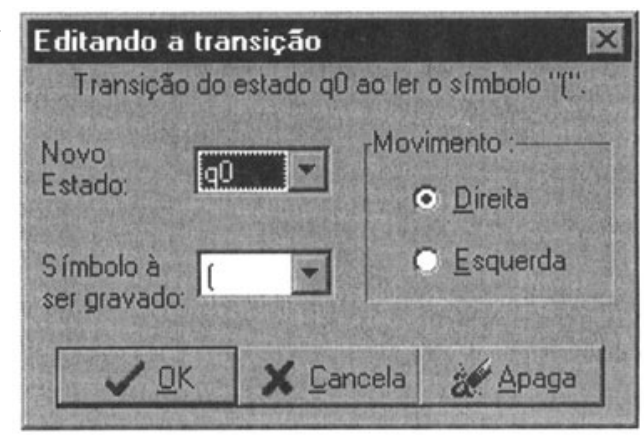

Figure 5. Editing Screen

This module is divided basically into three parts: machine definition, editing of the transition matrix (program) and execution. We can define the machine by introducing its symbol alphabets (entries and auxiliaries) in its states. The programming is achieved by editing the transitions as a matrix element. It is carried out by clicking the desired position (state $\mathrm{x}$ symbol) and by filling in all the necessary fields, as showed in Figure 5. Finally, there 
is the program's execution window, in which the user can enter the tape and execute his program, either through the step by step or through the uninterrupted form.

The actual state of the program and the position of the tape reader are represented by selecting the correspondent cell. There are options to be chosen, so that during the execution the simulator is able to show the last states of the tape, as well as to save in one file all the tape's contents that were created during each step of the computation.

The Editor module, with capacity to convert types, is the module that offers program editor facilities in both types of programs and allows the conversion of a program through graphs in a program through transition functions and vice-versa. It allows the editing of programs simultaneously in the two formats; that is, it makes the compatibility and the translation between formats and programs.

\section{NORMA REGISTER MACHINE SIMULATOR}

Norma (Number Theoretic Register Machine) is a universal machine defined by Bird (Bird 1976). The memory is composed of infinite registers and the entry and exit sets are non-null integers. The operations and tests defined by Norma are the most natural ones: to add and subtract a unit to the register and verify whether a register is zero. We can notice that in the definition of the subtraction over the A register, it is supposed that, when the A register is already null, the null value remains in $\mathrm{A}$.

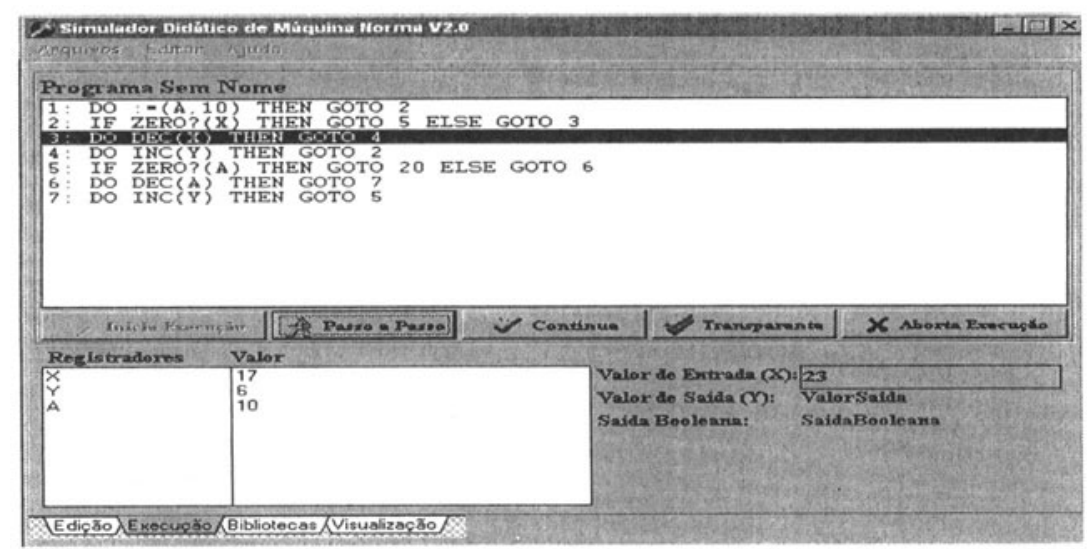

Figure 6. Norma program Editor 
The registers $\mathrm{X}$ and $\mathrm{Y}$ have special treatments. The entry function of Norma loads the $\mathrm{X}$ register with the entry number and starts all the other registers with zero. The Norma exit function withdraws the final content of the $\mathrm{Y}$ register as exit. Norma processes programs in a flowchart, showed as labelled instructions.

The simulator of the Norma register machine was developed in such a way that not only we can verify the functioning of Norma and the characteristics above, but we can also learn them with the assistance of tutorials and helps. The principal characteristics are as follows.

Registers. In order that we may simulate the functioning of this machine it has been defined that all registers will have a representation of 128 decimal digits, which implies the restriction that the major number is $10^{128}$;

Instructions format. To facilitate the implementation of the simulator, it has been defined that the instructions (operations and macros) would be represented in the following way: name $\left(O P_{1}, O P_{2}, \ldots, O P_{n}\right)$, where name is any word set of symbols (not containing numbers) and $O P_{1}, . ., O P_{n}$ are the registers involved. The basic operations are defined, respectively, as INC(A), DEC(A), ZERO?(A), to any A register.

Data entry. The data entry can be done in two ways: through flowcharts (written in a text box in the editing screen) or through the $\mathrm{X}$ entry value (which is introduced through a $\mathrm{n}$ editing box in the editing screen).

Macros. The macros are created in order to facilitate the development of programs. On the macro manipulation screen, all the information related to them must be defined. The previously defined macros can be re-utilised or consulted during the edition of a diagram, through boxes on the editing screen that contain the macros and their description.

Tutorial. The tutorial is divided into two parts: one part contains all the information about the NORMA register machine, and the other part contains all the information about the utilization of the simulator.

Conversion. It is possible to make the conversion of flowcharts into labeled instructions and vice-versa.

\section{POST MACHINE SIMULATOR}

Inside the context of the Theory of Computability, the formalism of the Post Machine (developed by the mathematician Emil Leon Post in 1936) is very important due to its equivalence to the most known Universal Machines (such as the Turing Machine). One of its main applications was the recognition of languages, necessary for the understanding and construction of a compiler. 


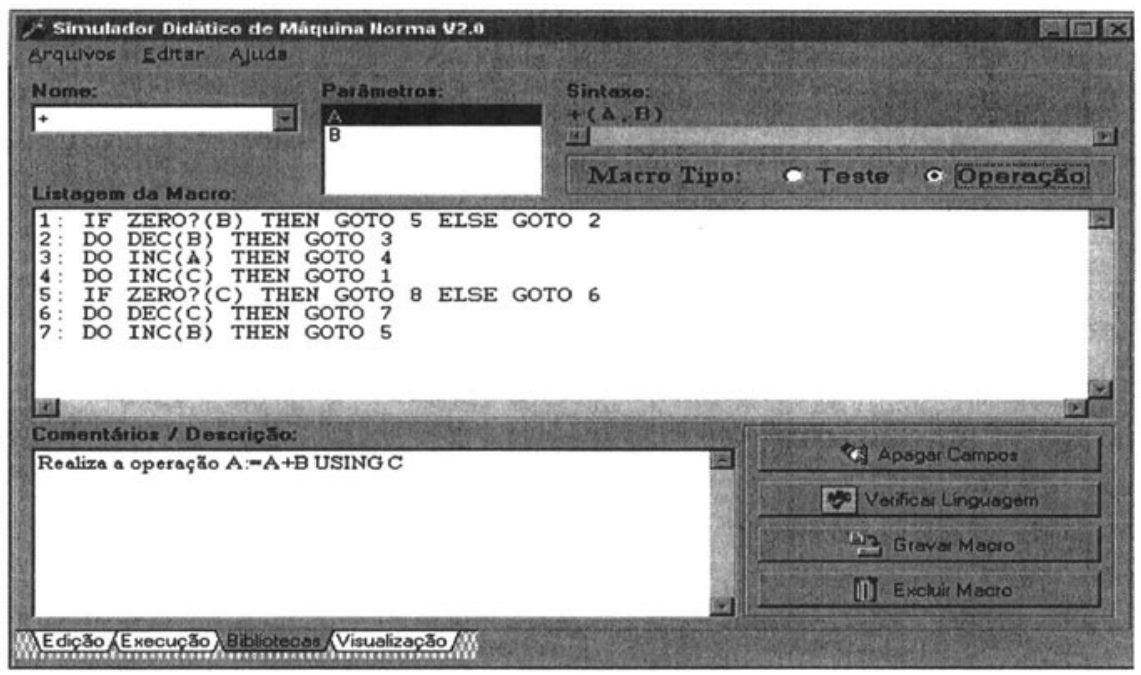

Figure 7. Macro Editors

The Post Machine is characterised by having a unique entry, exit and work memory structure, the queue type. It consists of two parts.

$\mathbf{X}$ variable. The mentioned structure, queue type, is used as memory, entry and exit. This variable does not have fixed size, or a limit. Its length is the length of a word stored in the time instant. In case the $\mathrm{X}$ does not have symbols, the entry is called empty and is represented by $\varepsilon$.

Program. It is a finite sequence of instructions, represented by diagram of flow, in which each vertices is an instruction.

The Simulator has two work environments: the editing environment and the executing environment. The editing environment is used for the definition of symbols to be used and for the entry of the function program in the form of a diagram composed of labels linked through transitions, in the same functions distinguished in the formal definition.

In the execution environment the editing screen is disabled and the computation of the word is made following the transitions connections with the respective label operations. The algorithm used is simple: from the first transition (start label) follow the transitions, and they carry out the operations indicated by the subsequent labels (attribution and test), until it finds a stop label (accepted/rejected). The computation, in this case, stops accepting or rejecting the entry word. 


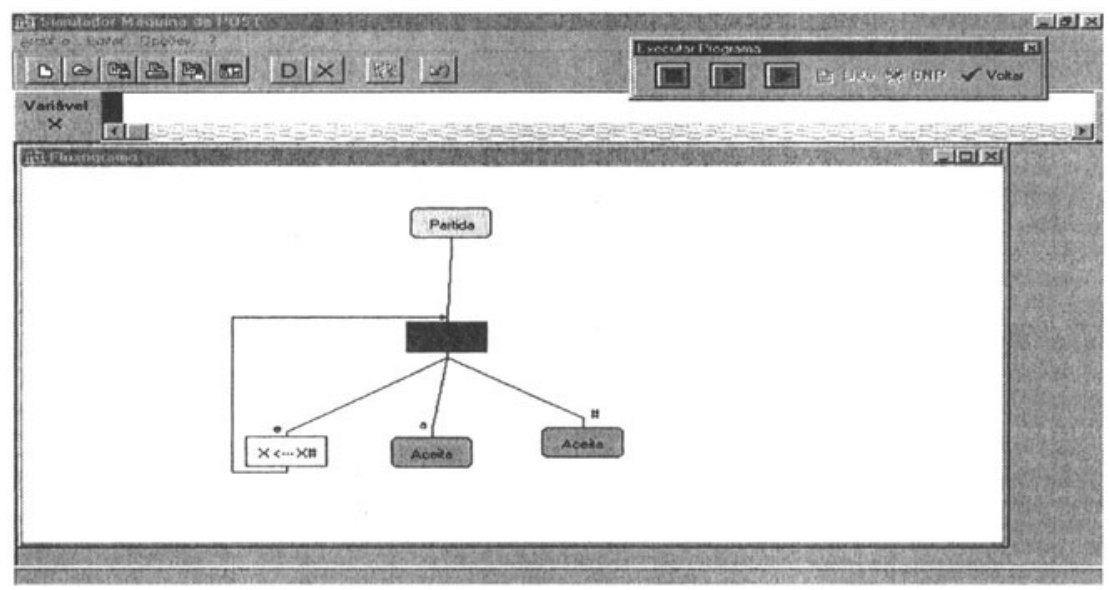

Figure 8. Execution Environment of the Post Simulator Machine

This tool is important mainly because of its pedagogical character, which helps in the comprehension of the concepts offered during the classes, providing teacher and students with a visual and interactive resource to work with this knowledge. Because it is small and portable software, the teaching resource can be used outside classroom, especially in the performance of exercises and works applied by the teacher.

\section{FINAL COMMENTS}

The simulator embraces two emphasis of the Turing Machine: one recognises words (Menezes 2000) and the other calculate functions (Diverio et al. 2000). The first one has in its definition two final states, which indicate whether the word has been accepted or rejected. The second one however has only one final state, which indicates whether the function's calculation has been successful or not. In both cases, if the machine stops in a state that is not defined as final, an error message is presented, and the execution is interrupted.

The simulators were initially developed with the purpose of being used in local environment, in which the software would be available in the laboratories of the Computer Science Institute. It aimed to stimulate the theoretical Computing teaching. However, they are being proposed as one of the modules of the remote teaching environment, which has been integrating teachers and students from the groups of Mathematics of Computation and High Performance Processing, Information Systems and Artificial Intelligence. 


\section{REFERENCES}

Bird, R. (1976) Programs and Machines - an introduction to the theory of computation. JohnWiley, London.

Diverio, T. A. and Francisco, B. R. T. (1996) Experiência do GMC no desenvolvimento de software Instrucional. In: Conferência Latinoamericana de Informática, XXII Clei 96, Santafé de Bogotá: CLEI, junho, pp.1003-1012, v.2.

Diverio, T. A., Tiarajú A. and Menezes P. F. B. (2000) Teoria da Computação: Máquinas Universais e Computabilidade. Sagra-Luzzato, Inst. Informática UFRGS, Porto Alegre.

Menezes, P. F. B. (2000) Linguagens Formais e Autômatos Finitos. Sagra-Luzzatto, Porto Alegre.

Moesch, T. F., Grandi, R. H., Lutz, F. and Diverio, T. A. (1998) Ferramentas de Apoio ao Ensino de Teoria da Computação. In: Conferencia Latino-Americana de Informática, Clei 98, Quito, Equador: CLEI, outubro. Vol. 1, pp. 13-24.

Mito, I. V. and Diverio, T. A. (2001) Foundations for Virtual Environment to Support Theory of Computation Teaching. In International Conference Intelligent Multimedia and Distance Education, ICIMADE '01, Fargo, USA, 1-3, June, 2001.

\section{BIOGRAPHY}

Tiarajú A. Diverio graduated in Mathematics for the UFRGS, and a Masters and Doctorate in Computer Science to the CPGCC (1986 and 1995). $\mathrm{He}$ is Professor and researcher of the Institute Computer Science at UFRGS. His areas of interest: include Processing of High Performance (Processing Vectorial, Parallel and Distributed and Parallel Mathematical Algorithms) and Informatics in Education (Educational Development of Software for Mathematics).

Ingrid de Vargas Mito graduated in Science Computer at UFRGS and is doing a Masters in Computer Science to the PPGC-UFRGS. Her areas of Interests are Artificial Intelligent, Agents and Informatics in Education.

Thiago Fernandes Moesch and Luis Franscisco Ramos Lima are graduates in Science Computer at UFRGS, with interests in Informatics in Education and E-Commerce. 